\title{
Longitudinal profile diagnostic scheme with subfemtosecond resolution for high-brightness electron beams
}

\author{
G. Andonian, ${ }^{1,2}$ E. Hemsing, ${ }^{1}$ D. Xiang, ${ }^{3}$ P. Musumeci, ${ }^{1}$ A. Murokh, ${ }^{2}$ S. Tochitsky, ${ }^{4}$ and J. B. Rosenzweig ${ }^{1}$ \\ ${ }^{1}$ Department of Physics and Astronomy, UCLA, Los Angeles, California 90095, USA \\ ${ }^{2}$ RadiaBeam Technologies, Santa Monica, California 90404, USA \\ ${ }^{3}$ SLAC National Accelerator Laboratory, Menlo Park, California 94025, USA \\ ${ }^{4}$ Department of Electrical Engineering, UCLA, Los Angeles, California 90095, USA
}

(Received 10 March 2011; published 13 July 2011)

\begin{abstract}
High-resolution measurement of the longitudinal profile of a relativistic electron beam is of utmost importance for linac based free-electron lasers and other advanced accelerator facilities that employ ultrashort bunches. In this paper, we investigate a novel scheme to measure ultrashort bunches (subpicosecond) with exceptional temporal resolution (hundreds of attoseconds) and dynamic range. The scheme employs two orthogonally oriented deflecting sections. The first imparts a short-wavelength (fast temporal resolution) horizontal angular modulation on the beam, while the second imparts a long-wavelength (slow) angular kick in the vertical dimension. Both modulations are observable on a standard downstream screen in the form of a streaked sinusoidal beam structure. We demonstrate, using scaled variables in a quasi-1D approximation, an expression for the temporal resolution of the scheme and apply it to a proofof-concept experiment at the UCLA Neptune high-brightness injector facility. The scheme is also investigated for application at the SLAC NLCTA facility, where we show that the subfemtosecond resolution is sufficient to resolve the temporal structure of the beam used in the echo-enabled free-electron laser. We employ beam simulations to verify the effect for typical Neptune and NLCTA parameter sets and demonstrate the feasibility of the concept.
\end{abstract}

DOI: 10.1103/PhysRevSTAB.14.072802

PACS numbers: 29.27.Fh, 41.75.Lx, 41.75.Jv

\section{INTRODUCTION}

Next generation free-electron lasers (FELs), such as the Linac Coherent Light Source (LCLS), and other advanced accelerator facilities require ultrashort pulses $(\sim$ few femtoseconds) with high peak currents $\left(\geq 10^{3} \mathrm{~A}\right.$ ) for successful operations. Such ultrashort pulses are achieved by chicane compressors or velocity bunching techniques [1] that produce high peak currents without compromising transverse emittance quality. The careful measurement of the overall bunch length and temporal profile of these pulses is critical for performance optimization and benchmarking to computational models. Reasonable longitudinal characterization of these short beams demands a resolution on the femtosecond scale or better, in order to study phenomena on the ultrashort length or ultrafast time scales such as the microbunching instability behind the observation of coherent optical transition radiation [2-4], the longitudinal beam properties of echo-enabled FELs [5], the properties of drivers for attosecond $\mathrm{x}$-ray production [6], or beam studies in single-spike self-amplified spontaneous emission FEL processes [7].

Published by the American Physical Society under the terms of the Creative Commons Attribution 3.0 License. Further distribution of this work must maintain attribution to the author $(s)$ and the published article's title, journal citation, and DOI.
Present-day bunch length diagnostics include a wide range of schemes including electro-optical techniques [8], rf deflecting cavities [9], rf zero-crossing methods [10], and the deconvolution of the frequency spectrum of emitted radiation sources [11,12]. These methods are well tested and robust, with resolutions reported as low as $<10$ fs. Alternative methods to achieve femtosecond level resolutions in literature aim at exploiting and manipulating beam phase space correlations $[13,14]$. In this paper, we describe a novel scheme for a longitudinal profile diagnostic that can readily achieve subfemtosecond resolution. The scheme exploits the interaction of an ultrashort beam with a high-power laser operating in a higher-order transverse Gaussian mode (e.g. $\mathrm{TEM}_{10}$ ) and a planar undulator resonant at the laser frequency. The combined laser/undulator interaction generates an angular modulation in the electron beam at the wavelength of the laser. For beams shorter than half the laser wavelength, $\lambda / 2$, the full temporal structure of the bunch is encoded into the horizontal angles and thus observable on a distant screen. For bunches longer than $\lambda / 2$ (which will be the primary focus of this work), the entire bunch is resolvable with another angular modulation, which is imparted on the beam in the vertical direction by means of an rf deflecting cavity. The end result is a "snakelike" pattern on the screen (see Fig. 1 for an experimental layout). The vertical length of the curve corresponds to the bunch length of the beam, while temporal features on the order of hundreds of attoseconds are 


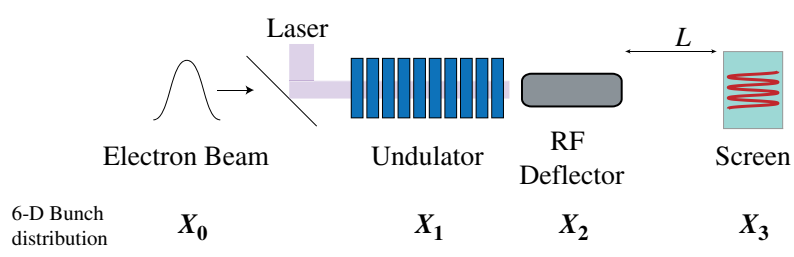

FIG. 1. Conceptual scheme for longitudinal profile diagnostic with sub-fs resolution. The undulator provides coupling between the laser mode and ultrashort electron bunch to impart an angular modulation. The deflecting cavity provides a vertical sweep in the orthogonal direction. The pattern is swept on a distant screen. The beam distributions at each element along the beam line are given by $X_{i}$, where $i=0,1,2,3$ corresponds to the $6 \mathrm{D}$ beam distribution (0) initially, (1) after the modulator, (2) after the deflector, and (3) at the screen, respectively.

resolvable along the sinusoidal curve with the appropriate choice of readily available optics and monitors. The entire scheme is compact and can be easily incorporated into a diagnostic line at any high-brightness facility that employs compressed beams, with an accompanying laser system.

\section{TRANSVERSE ENCODING OF TEMPORAL PROFILE AND GENERAL SCALING}

To get a sense of the characteristics of the system for some general scaling, we model the arrangement as three simple components: (1) the laser modulator (undulator), (2) the rf deflector, and (3) the drift to the diagnostic screen. Each performs a linear transformation on the $6 \mathrm{D}$ electron beam phase space, and for the purposes of generating simple analytical formulas, we assume the laser modulator and rf deflector are zero-length elements. The schematic is illustrated in Fig. 1 and the coordinate index numbers $(0,1,2,3)$ are used as subscripts to indicate location along the beam line. The initial e-beam distribution is given by the function $f_{0}=f_{0}\left(X_{0}\right)=$ $f_{0}\left(x_{0}, x_{0}^{\prime}, y_{0}, y_{0}^{\prime}, \eta_{0}, s_{0}\right)$, where $x_{0}$ and $y_{0}$ are the transverse spatial coordinates of an electron, $x_{0}^{\prime}=d x_{0} / d z$ and $y_{0}^{\prime}=$ $d y_{0} / d z$ are the transverse divergences, $\eta_{0}=\left(\gamma_{0}-\gamma\right) / \gamma$ is the relative energy deviation of an electron with energy $\gamma_{0}$ from the nominal e-beam energy $\gamma$, and $s_{0}=z-v_{0} t$ is the position of the electron in the moving frame.

The laser modulator is based on a concept investigated by Zholents and Zolotorev [15] and we follow their notation where applicable. In their study, a portion of the e-beam interacts with a few-cycle TEM $_{10}$ HermiteGaussian mode in a planar undulator (horizontal wiggle induced with vertical field orientation) for the production of attosecond pulses. In our scenario however, the entire ebeam is evenly modulated in energy and horizontal angle along the bunch length, so we simplify by assuming that the laser pulse length is much longer than the e-beam bunch length.
The electric field of the $\mathrm{TEM}_{10}$ mode is given by

$$
E_{x}(x, z, t) \simeq \frac{2 \sqrt{2} E_{0} x}{w_{R}\left(1+z^{2} / z_{R}^{2}\right)} \sin [k(z-c t)+\phi],
$$

where $E_{0}$ is the field amplitude, $\phi$ is the laser phase, $k=2 \pi / \lambda$ is the laser wave number, $\lambda$ is the laser wavelength, $w_{R}$ is the laser waist, and $z_{R}$ is Rayleigh range. In this expression, we have neglected the Gaussian transverse dependence of the field in the assumption that the laser waist is much larger than the transverse e-beam size. We refer to this as a quasi-1D approximation, where the field experienced by the e-beam is linear in $x$.

The normalized transverse velocity of an electron inside the undulator is given by

$$
\beta_{x}=-\frac{K}{\gamma} \sin \left(2 \pi z / \lambda_{u}\right),
$$

where the dimensionless undulator parameter is $K=$ $\left(e B_{0} \lambda_{u}\right) /\left(2 \pi m_{0} c\right), B_{0}$ is the peak magnetic field in the undulator, and $\lambda_{u}$ is the undulator period. The effect of the laser on the transverse motion is neglected in the limit that $K \gg e E_{0} \lambda /\left(2 \pi m_{0} c^{2}\right)$. The energy exchange between the electron and the fields is

$$
\frac{d \gamma}{d t}=\frac{e}{m_{0} c} E_{x} \beta_{x} .
$$

Combining Eqs. (1)-(3) (see Zholents and Zolotorev [15]) yields an expression for the change in relative energy $(\Delta \gamma / \gamma \ll 1)$ of each particle as a function of its initial horizontal $\left(x_{0}\right)$ and longitudinal $\left(s_{0}\right)$ coordinates within the bunch, after the undulator (denoted by the index 1 - see Fig. 1) near the resonant energy $\gamma_{r}^{2}=\frac{\lambda_{u}}{\lambda}\left(1+K^{2} / 2\right)$,

$$
\frac{\Delta \gamma}{\gamma}=\eta_{1}-\eta_{0}=A k x_{0} \cos \left(k s_{0}\right)
$$

where

$$
A=\frac{2 K}{\gamma^{2}} \sqrt{\frac{P_{L}}{P_{0}}}[J J] f\left(L_{u}, z_{R}, \nu\right),
$$

$P_{L}=E_{0}^{2} \pi w_{R}^{2} /\left(2 \mu_{0} c\right)$ is the laser power, $\mu_{0}$ is the permeability of free space, $P_{0}=I_{A} m c^{2} / e$ where $I_{A}$ is the Alfvén current, $\quad[J J]=J_{0}\left[K^{2} /\left(4+2 K^{2}\right)\right]-J_{1}\left[K^{2} /\left(4+2 K^{2}\right)\right]$, $J_{0}$ and $J_{1}$ are Bessel functions, and $f$ is a function that depends on the undulator length $\left(L_{u}\right)$, detuning $\nu=$ $2 L_{u} \lambda_{u}\left(1-\gamma / \gamma_{r}\right)$, and Rayleigh range $\left(z_{R}\right)$ of the laser. This function is typically peaked at about $\sim 3$ for all practical values and may include beam emittance effects as well.

Note that the electrons on axis are unmodulated. Then by using the relation [15]

$$
\frac{\partial}{\partial x}\left(\frac{\Delta \gamma}{\gamma}\right)=\frac{\partial}{\partial s} \Delta x^{\prime}
$$


the change in horizontal divergence in such an interaction is written as

$$
x_{1}^{\prime}-x_{0}^{\prime}=\Delta x^{\prime}=A \sin \left(k s_{0}+\phi\right) .
$$

An example of the energy and angular modulations imprinted on the e-beam at the modulator is shown in Fig. 2.

The modulated electron beam then enters the rf deflector, which has a peak voltage $V_{d}$ and wave number $k_{\mathrm{rf}}=$ $2 \pi / \lambda_{\mathrm{rf}}$. For a beam at the zero-crossing phase, the deflector performs an analogous transformation on each electron's vertical coordinate, $y$, but at a wavelength long compared to the longitudinal e-beam size: $y_{2}^{\prime}=$ $y_{1}^{\prime}+A_{\mathrm{rf}} k_{\mathrm{rf}} s_{1}$ and $\eta_{2}=\eta_{1}+A_{\mathrm{rf}} k_{\mathrm{rf}} y_{1}$, where

$$
A_{\mathrm{rf}}=\frac{e V_{d}}{\gamma m c^{2}}
$$

is the form of a simple rf deflector. More explicitly, the phase space coordinates at the deflector $\left(X_{2}\right)$ in terms of the initial coordinates are (using $s_{0}=s_{1}$ )

$$
\begin{aligned}
x_{2} & =x_{0}, \\
x_{2}^{\prime} & =x_{0}^{\prime}+A \sin k s_{0}, \\
y_{2} & =y_{0}, \\
y_{2}^{\prime} & =y_{0}^{\prime}+A_{\mathrm{rf}} k_{\mathrm{rf}} s_{0}, \\
s_{2} & =s_{0}, \\
\eta_{2} & =\eta_{0}+A k x_{0} \cos k s_{0}+A_{\mathrm{rf}} k_{\mathrm{rf}} y_{0} .
\end{aligned}
$$

We note that here we assume zero length between the undulator and deflector, for the transformations, which is a good approximation for the short drift. Finally, the subsequent drift of length $L$ maps the coordinates from the
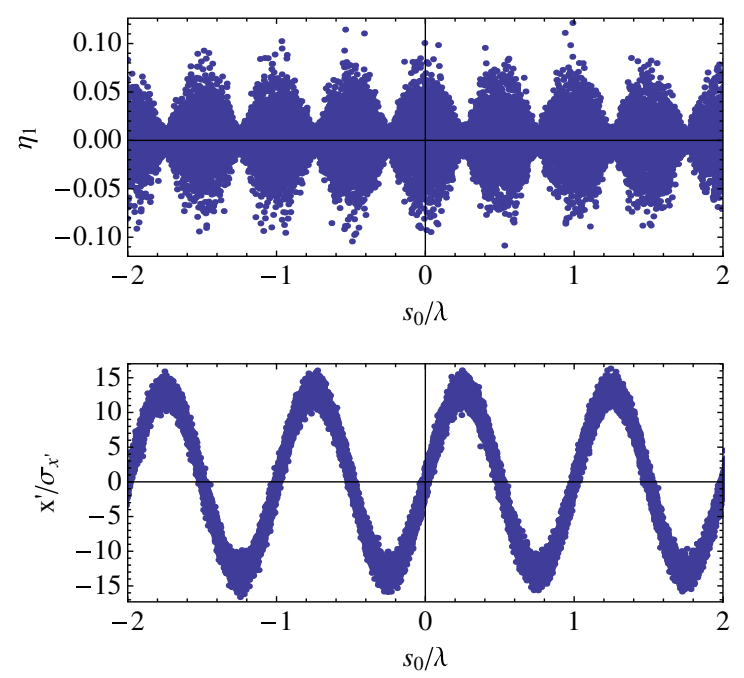

FIG. 2. Energy modulation of the beam (top) and horizontal angular modulation (bottom) after the laser interaction in the undulator calculated using Eq. (7). The longitudinal coordinates are normalized to the drive laser wavelength. deflector to the coordinates at the detector screen via $x_{3}=$ $x_{2}+L x_{2}^{\prime}, y_{3}=y_{2}+L y_{2}^{\prime}$, and $s_{3}=s_{2}+L \eta_{2} / \gamma^{2}$. With all contributions, the phase space coordinates at the detector screen, $X_{3}$, are then written in terms of the initial coordinates as

$$
\begin{aligned}
x_{3} & =x_{0}+L\left(x_{0}^{\prime}+A \sin k s_{0}\right), \\
x_{3}^{\prime} & =x_{0}^{\prime}+A \sin k s_{0}, \\
y_{3} & =y_{0}+L\left(y_{0}^{\prime}+A_{\mathrm{rf}} k_{\mathrm{rf}} s_{0}\right), \\
y_{3}^{\prime} & =y_{0}^{\prime}+A_{\mathrm{rf}} k_{\mathrm{rf}} s_{0}, \\
s_{3} & =s_{0}+\frac{L}{\gamma^{2}}\left(\eta_{0}+A k x_{0} \cos k s_{0}+A_{\mathrm{rf}} k_{\mathrm{rf}} y_{0}\right), \\
\eta_{3} & =\eta_{0}+A k x_{0} \cos k s_{0}+A_{\mathrm{rf}} k_{\mathrm{rf}} y_{0} .
\end{aligned}
$$

We note that Eq. (10) is a simple form that assumes zerolength elements for the undulator and deflector, and also assumes a zero-length drift between the undulator and deflector.

To assess the effect of the full transformation on the e-beam, we consider an uncorrelated initial six-dimensional electron distribution function at the modulator entrance:

$$
\begin{aligned}
f_{0}= & \frac{1}{(2 \pi)^{3} \sigma_{x}^{2} \sigma_{x^{\prime}}^{2} \sigma_{s} \sigma_{\eta}} \\
& \times \exp \left[-\frac{x_{0}^{2}+y_{0}^{2}}{2 \sigma_{x}^{2}}-\frac{x_{0}^{\prime 2}+y_{0}^{\prime 2}}{2 \sigma_{x^{\prime}}^{2}}-\frac{s_{0}^{2}}{2 \sigma_{s}^{2}}-\frac{\eta_{0}^{2}}{2 \sigma_{\eta}^{2}}\right],
\end{aligned}
$$

where we assume a round transverse beam $\left(\sigma_{x}=\sigma_{y}\right.$, $\sigma_{x^{\prime}}=\sigma_{y^{\prime}}$.

The bunch resolution of each individual beam line component (laser modulator, rf deflector) is set by the magnitude of the imprinted angular modulation, which must be larger than the intrinsic angles in the beam in order to resolve the structure. After passing through the rf deflector, the rms vertical angular size is given by

$$
\sigma_{y_{2}^{\prime}}^{2}=\int y_{2}^{\prime}\left(X_{0}\right)^{2} f_{0} d X_{0}=\sigma_{y^{\prime}}^{2}+A_{\mathrm{rf}}^{2} k_{\mathrm{rf}}^{2} \sigma_{s}^{2} .
$$

The shortest longitudinal structure that can easily be resolved in the vertical direction is therefore given by $\Delta s_{\mathrm{rf}}=$ $\sigma_{y^{\prime}} / A_{\mathrm{rf}} k_{\mathrm{rf}}$. This quantity must be smaller than $\lambda$ in the laser modulator in order to distinguish between particles in adjacent laser cycles. The resolution of the laser modulator (LM) is calculated analogously, and is given by $\Delta s_{\mathrm{LM}}=$ $\sigma_{x^{\prime}} / A k$. In modern high-power laser-modulator systems, $A k$ can be made much larger than $A_{\mathrm{rf}} k_{\mathrm{rf}}$, so the effective temporal resolution of the orthogonal deflecting cavities is dominated by the laser modulator:

$$
\Delta t_{\mathrm{LM}}=\frac{\epsilon_{n, x}}{\gamma \sigma_{x} A c k},
$$

where $\epsilon_{n, x}$ is the normalized horizontal emittance of the beam, and we have assumed no position-angle correlation in the beam. This is a significant improvement over the 


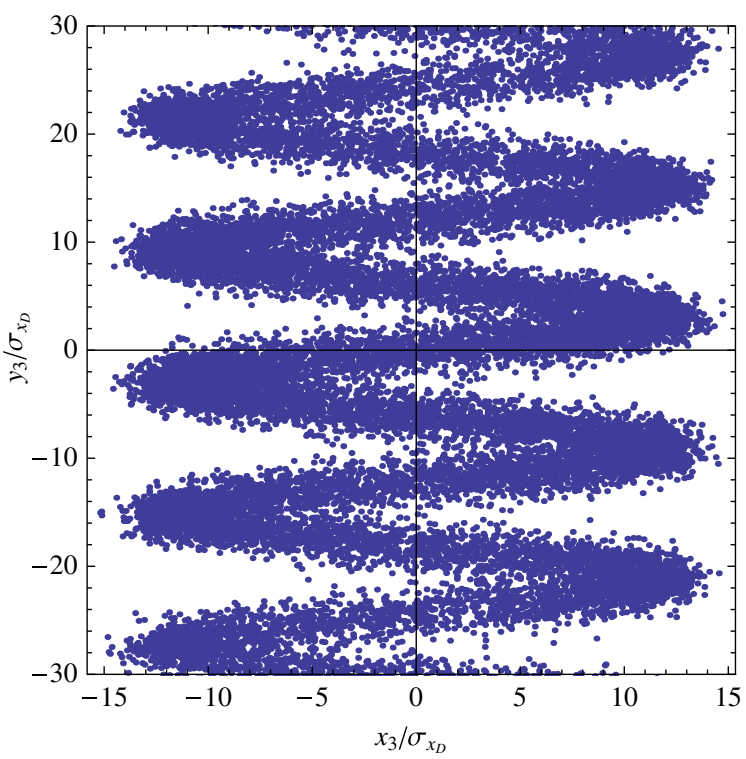

FIG. 3. Depiction of the transverse distribution on the screen calculated from the analytical expressions of Eq. (10). The axes are normalized to $\sigma_{x_{D}}$, the unperturbed beam size at the detector due only to the drift (i.e. with the laser and deflector turned off).

temporal resolution of the rf deflector alone. An example screen distribution is shown in Fig. 3, where the sinusoidal pattern is clearly visible.

Using the transformations in Eq. (10), it is useful to examine the e-beam distribution at the screen in terms of $s_{0}$ :

$$
\begin{aligned}
f_{0}\left(x_{3}, y_{3}, s_{0}\right)= & \frac{1}{(2 \pi)^{3 / 2} \sigma_{x_{D}}^{2} \sigma_{s}} \exp \left[-\frac{\left(x_{3}-A L \sin k s_{0}\right)^{2}}{2 \sigma_{x_{D}}^{2}}\right. \\
& \left.-\frac{\left(y_{3}-A_{\mathrm{rf}} k_{\mathrm{rf}} L s_{0}\right)^{2}}{2 \sigma_{x_{D}}^{2}}-\frac{s_{0}^{2}}{2 \sigma_{s}^{2}}\right]
\end{aligned}
$$

where $\sigma_{x_{D}}^{2}=\sigma_{x}^{2}+L^{2} \sigma_{x^{\prime}}^{2}$ is the unperturbed mean squared beam size at the detector due only to the drift section. From Eq. (14) the horizontal and vertical rms beam sizes at the detector are given by

$$
\begin{aligned}
& \sigma_{x_{3}}^{2}=\sigma_{x_{D}}^{2}+\frac{A^{2} L^{2}}{2}\left(1-e^{-2 k^{2} \sigma_{s}^{2}}\right), \\
& \sigma_{y_{3}}^{2}=\sigma_{x_{D}}^{2}+A_{\mathrm{rf}}^{2} k_{\mathrm{rf}}^{2} L^{2} \sigma_{s}^{2} .
\end{aligned}
$$

If $k \sigma_{s} \gg 1$, the horizontal beam size, $\sigma_{x_{3}}$, is independent of the bunch length. Note the similarity between the form of $\sigma_{x_{3}}$ and $\sigma_{y_{3}}$ in the opposite limit $\left(k \sigma_{s} \ll 1\right)$ where both upstream sections act as simple linear deflectors, as expected.

There are a few fundamental constraints on the system to serve as an effective longitudinal profile diagnostic. First, for simultaneous resolution of the horizontal sinusoid and the vertically streaked structure at the detector, the imposed beam deflections should be larger than the unperturbed beam size at the screen, so, for a round beam,

$$
\frac{A_{\mathrm{rf}} k_{\mathrm{rf}} L \sigma_{s}}{\sigma_{x_{D}}} \gg 1 \text { and } \frac{A L}{\sqrt{2} \sigma_{x_{D}}} \gg 1
$$

These constraints make it necessary in certain cases to employ collimators to reduce the e-beam transverse size. Finally, each of these values must be less than the screen dimensions if one desires to sample the entire beam on the detector.

The main advantages of this diagnostic scheme are the increased absolute resolution and the increase in dynamic range (DR) available for discerning sub-bunch-length structure. Here, we quantify the gain in DR in terms of scaled variables. Consider the distribution at the detector screen [Eq. (14)] which is peaked along a curve given by

$$
x_{3}\left(y_{3}\right)=A L \sin \left(\frac{2 \pi y_{3}}{\lambda_{y_{3}}}\right),
$$

and has a vertical wavelength of

$$
\lambda_{y_{3}}=\frac{2 \pi k_{\mathrm{rf}} A_{\mathrm{rf}} L}{k} .
$$

The peak-to-peak amplitude of the horizontal deflection imparted by the $\mathrm{TEM}_{10}$ mode is $2 A L$. The path length the sinusoidal beam traces on the screen is found from the expression $d \xi^{2}=d x_{3}^{2}+d y_{3}^{2}$, or $\xi=\int d y_{3} \times$ $\sqrt{1+\Gamma^{2} \cos ^{2}\left(2 \pi y_{3} / \lambda_{y_{3}}\right)}$ using (17) where $\Gamma=A k / A_{\mathrm{rf}} k_{\mathrm{rf}}$. Integrating from $-\sigma_{y_{3}} / 2$ to $\sigma_{y_{3}} / 2$ gives the path length of the e-beam subtended on the screen over the vertical rms image size. This gives

$$
\xi(\Gamma)=\frac{\lambda_{y_{3}}}{\pi} \sqrt{1+\Gamma^{2}} E\left[\frac{\pi \sigma_{y_{3}}}{\lambda_{y_{3}}}, \frac{\Gamma^{2}}{1+\Gamma^{2}}\right],
$$

where $E$ is the elliptic integral of the second kind. Here, we define the dynamic range DR of the system as the ratio of path length trace on the screen $\xi$ to the resolution of the system for each component. With the laser field off, the path length is $\xi(0)=\sigma_{y_{3}}$ as one would expect from a simple $\mathrm{rf}$ deflector. If the vertical deflection is large compared to $\sigma_{x_{D}}$ at the detector, the dynamic range from the $\mathrm{rf}$ deflector alone is $\mathrm{DR}_{\mathrm{rf}}=\xi(0) / \Delta z_{\mathrm{rf}} \simeq$ $\left(A_{\mathrm{rf}} k_{\mathrm{rf}}\right)^{2} L \gamma \sigma_{s} \sigma_{x} / \epsilon_{n, x}$. With the laser modulator also turned on and in the desirable limit that $\Gamma \gg 1$, the path length is $\xi \simeq(0.55-1) \sigma_{y_{3}} \Gamma$, with the exact proportionality dependent on the magnitude of $\lambda_{y_{3}} / \pi \sigma_{y_{3}} \simeq 2 / k \sigma_{s}$. With $\Gamma \gg 1$ the resolution is dominated by the laser modulator, and the dynamic range of the system is $\mathrm{DR}=\xi / \Delta z_{\mathrm{LM}} \simeq$ $(A k)^{2} L \gamma \sigma_{s} \sigma_{x} / \epsilon_{n, x}$. Taking the ratio we find

$$
\mathrm{DR} / \mathrm{DR}_{\mathrm{rf}} \simeq \Gamma^{2} \text {. }
$$

The laser-modulator section thus provides a quadratic improvement in the dynamic range of the overall system for characterization of the temporal bunch structure for $\Gamma \gg 1$, provided that the entire beam can be imaged. 


\section{BUNCH PROFILE DIAGNOSTIC}

Now we consider specific examples of the diagnostic scheme with simulations that demonstrate the efficacy of temporal profile resolution. First, the UCLA Neptune case is shown as a proof-of-concept experiment. Then we consider the echo-enabled FEL case at SLAC, where there is a direct need to resolve structures on the sub-fs scale.

\section{UCLA Neptune high-brightness photoinjector}

The UCLA Neptune advanced accelerator facility employs a high-brightness photoinjector and has a rich history in the study of electron beam-laser interactions $[16,17]$. The beam parameters for the facility include an energy of $13 \mathrm{MeV}$, relative uncorrelated energy spread of $5 \times 10^{-3}$, and nominal normalized emittance of $\epsilon_{n, x}=$ $\epsilon_{n, y} \simeq 1 \mathrm{~mm} \mathrm{mrad}$ at an operating charge of $Q=500 \mathrm{pC}$. The facility also has a high-power $\mathrm{CO}_{2}$ laser $(\lambda=10.6 \mu \mathrm{m})$ which has the ability to produce and amplify higher-order transverse Gaussian modes (e.g. TEM $_{10}$ ) to near GW power levels.

For the current scheme we explore a comparable parameter set (see Table I) in analytical and simulation studies presented here. The near-resonance condition for the $\mathrm{CO}_{2}$ laser $(\lambda=10.6 \mu \mathrm{m})$ is satisfied with a ten period undulator $\left(N_{u}=10\right)$ of peak field $B_{0}=1.02 \mathrm{~T}$, and period $\lambda_{u}=9 \mathrm{~mm}$, or $K=0.855$, such as the undulator described in Ref. [18]. With a laser waist of $w_{R}=250 \mu \mathrm{m}$ this yields a value of $f=2.3$ for the function as defined in Ref. [5], with an operational beam energy of $12.9 \mathrm{MeV}$. In order to satisfy the constraints of Eq. (16), the e-beam is trimmed using a circular collimator of aperture $180 \mu \mathrm{m}$ before the laser-modulator section. The beam transmitted through the collimator is round with $\sigma_{x} \simeq 45 \mu \mathrm{m}$ and has an effective emittance approximately one-tenth of the nominal value $\epsilon_{n, x}=\epsilon_{n, y} \simeq 0.1 \mathrm{~mm}$ mrad. The temporal resolution, for a laser power of $P_{L}=300 \mathrm{MW}$, given by Eq. (13), is $\Delta t_{\mathrm{LM}} \simeq 330$ as.

For the Neptune UCLA parameters, an X-band deflecting cavity provides the appropriate working parameter set $\left(\lambda_{\mathrm{rf}}=2.6 \mathrm{~cm}, V_{d}=6 \mathrm{MV}\right)$ for this application. This $\mathrm{X}$-band deflector provides the necessary vertical kick to

TABLE I. Simulation parameters for the UCLA Neptune case.

\begin{tabular}{lcc}
\hline \hline Beam energy & $E$ & $12.9 \mathrm{MeV}$ \\
Normalized emittance & $\epsilon_{n}$ & $1.0 \mathrm{~mm} \mathrm{mrad}$ \\
Energy spread & $\sigma_{\gamma}$ & $5 \times 10^{-3}$ \\
Undulator peak field & $B_{0}$ & $1.02 \mathrm{~T}$ \\
Undulator period & $\lambda_{u}$ & $9 \mathrm{~mm}$ \\
Undulator length & $L_{u}$ & $9 \mathrm{~cm}$ \\
Undulator parameter & $K$ & 0.855 \\
Laser wavelength & $\lambda$ & $10.6 \mu \mathrm{m}$ \\
Laser waist & $w_{R}$ & $250 \mu \mathrm{m}$ \\
Laser power & $P_{L}$ & $300 \mathrm{MW}$ \\
\hline \hline
\end{tabular}

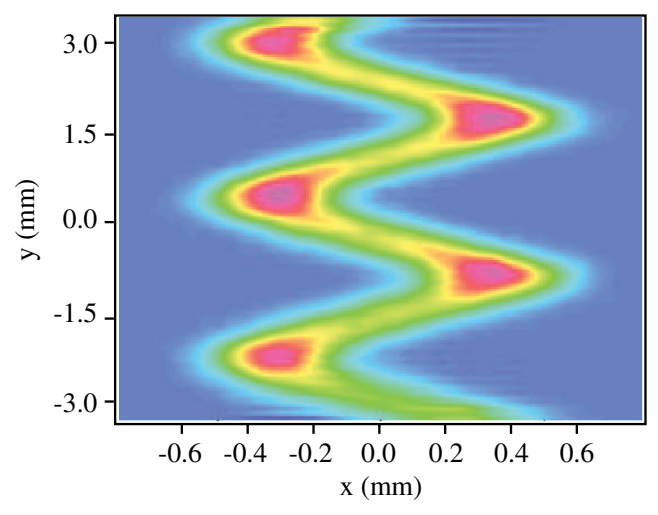

FIG. 4. ELEGANT simulation of the transverse profile at the end of the diagnostic line using Neptune parameters. The horizontal modulation and vertical streak are provided by the laser modulator and the deflecting cavity, respectively.

resolve the angular sweeps of the beam on a screen placed $L=1 \mathrm{~m}$ away.

Subsequent ELEGANT [19] simulations of the diagnostic line are used to account for all the effects. Optimized parameter sets show that indeed that sub-bunch-length structure is resolvable with the parameters above. The main tuning parameters include laser waist, and e-beam spot size (performed by addition of a round collimator prior to the laser modulator). Figure 4 shows a small part of the simulated detector screen after the beam passes through the rf deflector and a $1 \mathrm{~m}$ drift. We note that simulations show that $\sim 10 \mathrm{pC}$ of charge is transmitted through the collimator to the screen using the nominal Neptune parameters, and is readily resolved using standard screens like yttrium aluminium garnet (YAG) or phosphor. For completeness it is worth mentioning that, if the entire beam is imaged on the screen, the calculated dynamic range as defined above is $\mathrm{DR} \simeq 10^{5}$ for this parameter set.

Although the quasi-1D analytical formalism describes the effects well, we note that further optimization of the scheme requires minor deviation from some basic assumptions used in the analytical treatment. Specifically, 3D effects inside the undulator become important when the full e-beam size is on the same order as the laser waist size. In this case, the amplitude of the angular modulation calculated from the quasi-1D analysis is about $40 \%$ greater than that observed in numerical simulations, which also showed some smearing effects in the angular modulation due to detuning within the beam for edge particles also affected by the radius of curvature of the laser wave fronts. For the initial treatment, the beam size was considered much smaller than the laser waist, and when simulations were run in the $1 \mathrm{D}$ limit $\left(\sigma_{s} \ll w_{R} / \sqrt{2}, L_{u} \ll z_{r}\right)$, there was excellent quantitative agreement.

\section{ECHO-ENABLED FEL AT SLAC NLCTA}

A proof-of-principle echo-enabled harmonic generation (EEHG) experiment [5] has been conducted at the Next 
Linear Collider Test Accelerator (NLCTA) at SLAC. In the experiment, the electron beam is first modulated by a $795 \mathrm{~nm}$ laser, and then sent through a chicane with large momentum compaction. After being modulated again by a $1590 \mathrm{~nm}$ laser, density modulations at shorter wavelengths are generated after passage through a second chicane. The density modulation is confirmed by measuring the coherent radiation at the harmonic frequency of the seed lasers. But, because the coherent radiation at the harmonic frequency can be generated by the effect of each laser individually, it is not trivial to identify whether the coherent radiation from the e-beam is generated from the interplay of the two lasers, or by the individual laser. In the experiment, this issue is resolved indirectly by giving the e-beam a significant energy chirp, so that the EEHG signal and the harmonic signal generated by each individual laser shifts to different wavelengths.

However, the most direct way to distinguish the signals is to measure the beam density modulation in the time domain. Simulations show that, for the density modulation generated from the $1590 \mathrm{~nm}$ laser alone, only one current bump within each laser wavelength is generated (left plot of Fig. 5), while in EEHG multiple current bumps are present within each wavelength (right plot of Fig. 5) due to the shredding of the phase space in the first strong chicane. Because the distribution is periodic, only the phase space within two wavelengths is shown in Fig. 5. Direct visualization of the individual microbunches, which requires sub-fs resolution, not only provides further solid evidence of EEHG but also allows for a more quantitative study of the physics associated with harmonic generation.

The diagnostic scheme presented here may provide superior resolution and the ability to visualize the microbunch structure for the EEHG experiment. The beam and laser parameters used in ELEGANT simulations are listed in Table II.

In the simulations, we again assume an upright phase ellipse at the undulator (uncorrelated transverse position and angle coordinates). The beam horizontal and vertical beta functions are assumed to be both $40 \mathrm{~m}$ in the undulator and rf cavity, corresponding to an rms beam size of
TABLE II. Beam and laser parameters used in the NLCTA simulation.

\begin{tabular}{lcc}
\hline \hline Beam energy & $E$ & $120 \mathrm{MeV}$ \\
Normalized emittance & $\epsilon_{n}$ & $1 \mathrm{~mm} \mathrm{mrad}$ \\
Energy spread & $\sigma_{\gamma}$ & $1 \times 10^{-4}$ \\
Undulator peak field & $B_{0}$ & $1.075 \mathrm{~T}$ \\
Undulator period & $\lambda_{u}$ & $6 \mathrm{~cm}$ \\
Undulator length & $L_{u}$ & $18 \mathrm{~cm}$ \\
Undulator parameter & $K$ & 6.0 \\
Laser wavelength & $\lambda$ & $10.6 \mu \mathrm{m}$ \\
Laser power & $P_{L}$ & $500 \mathrm{GW}$ \\
Laser waist & $w_{R}$ & $1 \mathrm{~mm}$ \\
\hline \hline
\end{tabular}

$\sigma_{x}=410 \mu \mathrm{m}$ and rms divergence of $\sigma_{x^{\prime}}=10.3 \mu \mathrm{rad}$ in both planes. An X-band transverse cavity with voltage of $V_{d}=8 \mathrm{MV}$ is used to deflect the beam in the vertical direction. The rf cavity provides a deflection strength of $A_{\mathrm{rf}} k_{\mathrm{rf}} \simeq 16 \mathrm{~m}^{-1}$. The corresponding temporal resolution for the rf deflector is $\Delta t_{\mathrm{rf}}=\epsilon_{n, y} /\left(\gamma \sigma_{y} A_{\mathrm{rf}} c k_{\mathrm{rf}}\right) \approx 2.1 \mathrm{fs}$, sufficient to streak the particles from different laser cycles to different vertical positions. A $500 \mathrm{GW} \mathrm{CO}_{2}$ laser is used in our simulation to provide a maximal horizontal deflection of $250 \mu \mathrm{rad}$ in order to resolve the sub-fs fine structures. For the laser modulator, the maximal deflection is achieved at the zero-crossing phase with a deflection strength of $A k \simeq 150 \mathrm{~m}^{-1}$, and the corresponding temporal resolution is $\Delta t_{\mathrm{LM}} \simeq 230$ as. The modulation is observed in a downstream screen. Here, instead of a simple drift, several quadrupoles are used to provide a $\pi / 2$-phase advance between the rf deflector and the observation screen, in both planes, to convert angle to position at the screen. The simulated transverse beam profile on the screen is shown in Fig. 6 where one can see that the microbunches of Fig. 5 are clearly resolved.

We note that microbunches are only resolved when a $100 \mu \mathrm{m}$ collimator is used at the entrance to the undulator to reduce the horizontal beam size and, effectively, the emittance. As seen from Eq. (4), the particles that experience larger angular deflections also acquire an increased energy spread which, together with the momentum
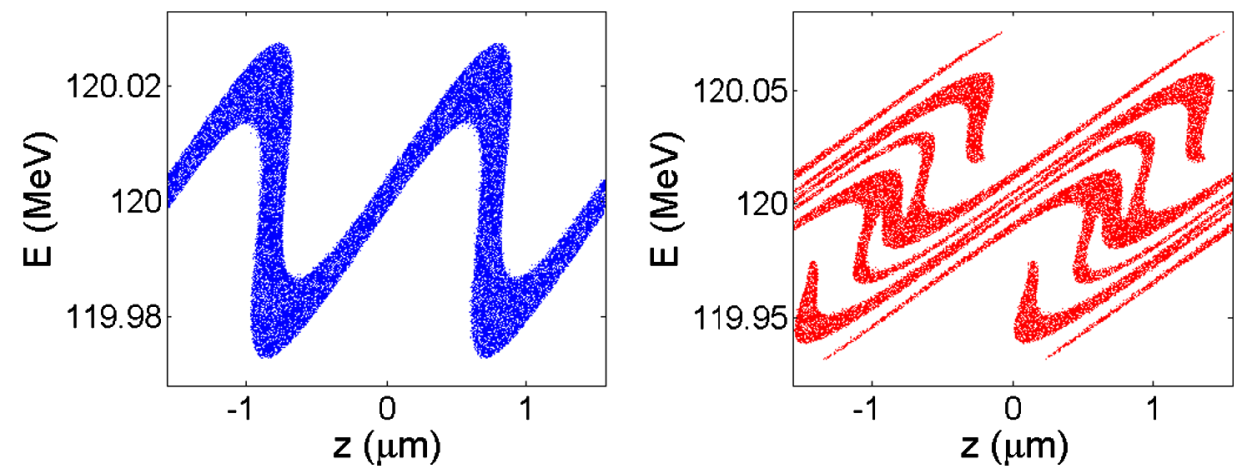

FIG. 5. Beam longitudinal phase space at the exit of the second chicane for the case when beam density modulation is generated by the $1590 \mathrm{~nm}$ laser alone (left) and that by the interplay of the two lasers (right). 

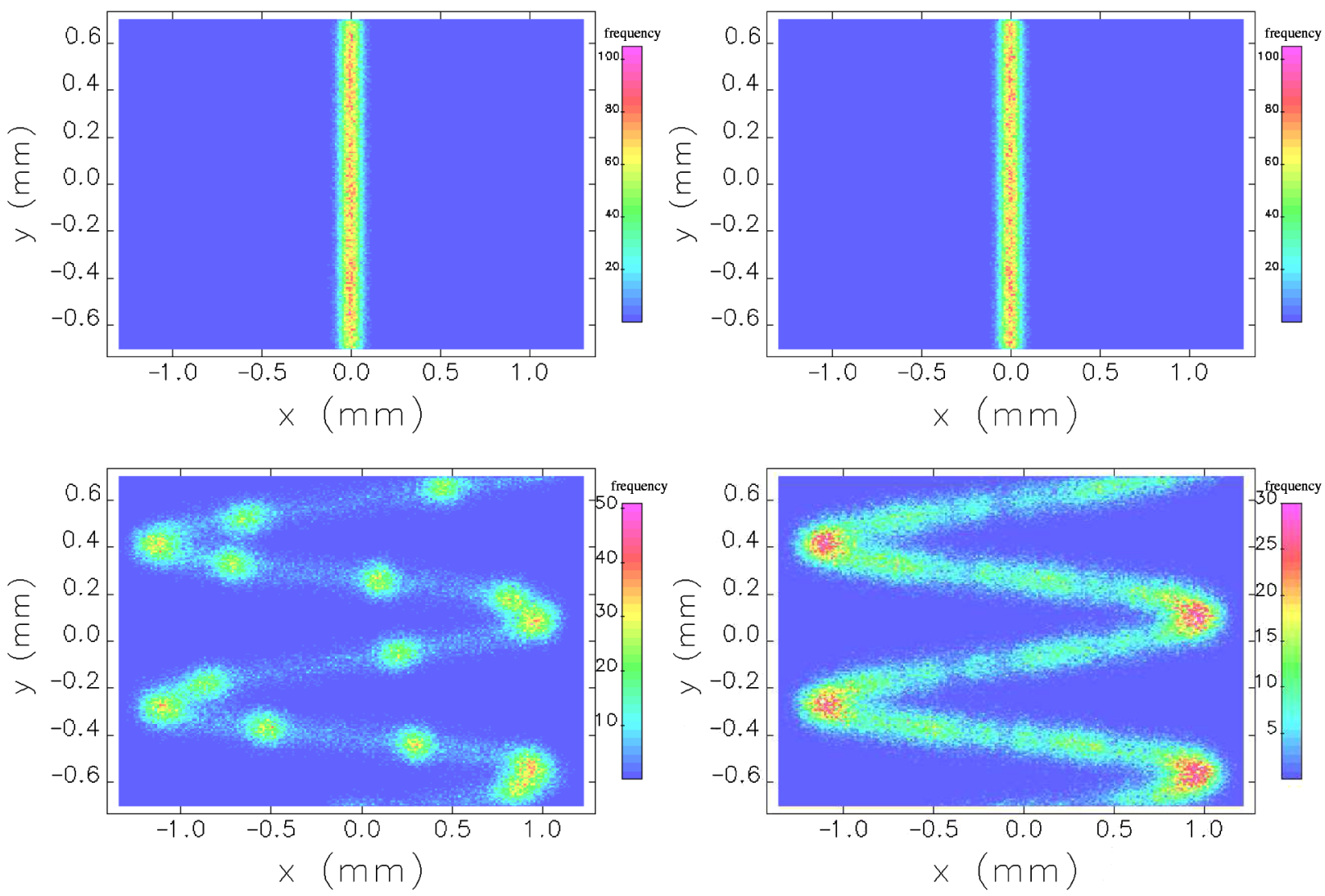

FIG. 6. Transverse beam profile at the screen for the case when the beam density modulation is generated by the $1590 \mathrm{~nm}$ laser individually (left) and that when the density modulation is generated by the interplay of the two lasers (right). The top figures show these cases when the deflector is powered on but the laser modulator (undulator) is turned off. The bottom figures illustrate the enhanced resolution when the laser modulator is turned on.

compaction of the undulator, tends to wash out fine structures. The effect is maximal at the turning points of the transverse distribution for particles with maximal angular deflection. Thus, without the collimator, the energy spread growth for the particles with maximal deflection in the EEHG scenario is $\sigma_{\Delta y / \gamma}=A k \sigma_{x} \simeq 6 \%$. Note the undulator has a momentum compaction of $R_{56}=2 N_{u} \lambda$. For the above case, this limits the resolution to $R_{56} \sigma_{\Delta \gamma / \gamma} / 2 \approx$ $1.9 \mu \mathrm{m}$, or about $6 \mathrm{fs}$, for maximally deflected particles at the turning points of the sinusoidal curve. With the $100 \mu \mathrm{m}$ collimator, the induced energy spread is reduced to $4.3 \times 10^{-3}$, corresponding to a temporal resolution of 450 attoseconds, sufficient to resolve the microbunches in EEHG across the entire curve.

Finally, the parameters used in our simulations and calculations are representative, rather than fully optimized design sets. A more careful optimization (i.e. reducing the number of periods of the undulator, reducing the aperture of the collimator, etc.) may easily lead to further improvements of the resolution.

\section{CONCLUSIONS}

The interaction between a high-brightness electron beam and high-power laser operating in a higher-order transverse mode in an undulator provides an angulartemporal correlation that is directly observable using standard imaging techniques. The use of this scheme as a longitudinal diagnostic has been studied and sub-fs temporal resolutions have been demonstrated using simulations, for two case studies. For a proof-of-concept experiment at the UCLA Neptune high-brightness injector, the expected resolution is on the order of $\sim 600$ attoseconds. Such a scheme has also been studied for use at the SLAC NLCTA facility, where the fine longitudinal structure of the beam used in the echo-enabled FEL is resolvable using this technique with a resolution of 450 attoseconds. Depending on beam line focusing elements and emittance, the diagnostic can be made compact (with total length $\sim 1 \mathrm{~m}$ ) and is easily incorporable into any present high-brightness facility or along a dedicated diagnostic line.

The presented scheme has some practical limitations that deserve comment and further clarification. First, the quoted resolutions for the diagnostic screen are not uniform along the bunch due to the sinusoidal modulation imposed by the undulator. The resolution is reduced at the turning points along the curve which may have implications for retrieval of complex bunch profiles in a single 
shot. For proper longitudinal resolution of the entire bunch, the curve can be scanned as a function of phase between the beam arrival time and the laser pulse $\phi$ of Eq. (1) to shift the beam away from these "low resolution" limits. A shift of $\phi=\pi / 2$ is necessary to move the portion of the beam from the "low resolution" region (i.e. the turning points along the curve) to the "high-resolution" region (i.e. the near-linear portion of the curve). A system scan using multiple shots at different phases (which may incidentally occur due to the inherent jitter in the system) will allow for fruitful data collection and subsequent full bunch profile reconstruction. Second, the scheme requires advanced specifications for the modulator driver (laser). Specifically, the $\mathrm{CO}_{2}$ laser is selected for the case studies due to its relatively long wavelength and high power, which are needed to provide the necessary angular modulation to achieve sub-fs resolution on low-mid energy beams, as evidenced in Eq. (7). Gigawatt class $\mathrm{CO}_{2}$ lasers have been successfully employed in electron beam interactions $[16,17,20]$, leading to the extension and the availability of terawatt-class $\mathrm{CO}_{2}$ lasers for high-brightness beam applications, shown in recent literature [21]. However, as such systems are elaborate and expensive, only a few facilities possess such capabilities, limiting the applicability of this specific scheme. For higher energy beams, the components of the scheme for comparable resolutions may require further efforts in specific technologies (i.e. long-period undulators, high-power lasers). Finally, the assumption of zero-length elements and negligible drifts between elements is useful for presenting an analytic expression of the beam transformations, but a full design requires careful reconsideration of these assumptions and detailed simulation studies to develop a realistic diagnostic.

In summary, the scheme proposed here is a diagnostic of the longitudinal bunch profile and provides unprecedented resolution useful for high quality operations, and in cases such as the EEHG experiment, necessary to unambiguously assert the fine structure of the studied effect.

\section{ACKNOWLEDGMENTS}

This work supported by U.S. DOE Office of Science SBIR Award No. DE-SC0004462. The work of D. Xiang was supported by the U.S. DOE under Contract No. DE-AC02-76SF00515.
[1] X. Wang, X. Qiu, and I. Ben-Zvi, Phys. Rev. E 54, R3121 (1996).

[2] H. Loos et al., Report No. SLACPUB-13395, 2008.

[3] A. H. Lumpkin, R. J. Dejus, and N. S. Sereno, Phys. Rev. ST Accel. Beams 12, 040704 (2009).

[4] S. Wesch, C. Behrens, B. Schmidt, and P. Schmuser, in Proceedings of the 31st International Free Electron Laser Conference (FEL 09), Liverpool, UK (STFC Daresbury Laboratory, Warrington, 2009), p. 619.

[5] D. Xiang et al., Phys. Rev. Lett. 105, 114801 (2010).

[6] Y. Ding, Z. Huang, D. Ratner, P. Bucksbaum, and H. Merdji, Phys. Rev. ST Accel. Beams 12, 060703 (2009).

[7] J. Rosenzweig et al., Nucl. Instrum. Methods Phys. Res., Sect. A 593, 39 (2008).

[8] I. Wilke, A. M. MacLeod, W. A. Gillespie, G. Berden, G. M. H. Knippels, and A. F. G. van der Meer, Phys. Rev. Lett. 88, 124801 (2002).

[9] R. Akre et al., in Proceedings of the 19th Particle Accelerator Conference, Chicago, Illinois, 2001 (IEEE, Piscataway, NJ, 2001), p. 2353.

[10] G. Krafft and J. Denard, in Proceedings of the Tenth Beam Instrumentation Workshop, Upton, New York (American Institute of Physics, New York, 2002), Vol. 648, p. 118.

[11] U. Happek, A. Sievers, and E. Blum, Phys. Rev. Lett. 67, 2962 (1991).

[12] G. Andonian et al., Phys. Rev. ST Accel. Beams 12, 030701 (2009).

[13] D. Xiang and Y. Ding, Phys. Rev. ST Accel. Beams 13, 094001 (2010).

[14] Z. Huang, K. Bane, Y. Ding, and P. Emma, Phys. Rev. ST Accel. Beams 13, 092801 (2010).

[15] A. Zholents and M. Zolotorev, New J. Phys. 10, 025005 (2008).

[16] S. Y. Tochitsky, O. B. Williams, P. Musumeci, C. Sung, D. J. Haberberger, A. M. Cook, J. B. Rosenzweig, and C. Joshi, Phys. Rev. ST Accel. Beams 12, 050703 (2009).

[17] P. Musumeci et al., Phys. Rev. Lett. 94, 154801 (2005).

[18] F. O'Shea, G. Marcus, J. Rosenzweig, M. Scheer, J. Bahrdt, R. Weingartner, A. Gauup, and F. Gruner, Phys. Rev. ST Accel. Beams 13, 070702 (2010).

[19] M. Borland, APS Technical Report No. LS-287, 2000.

[20] W. Kimura, G. Kim, R. Romea, L. Steinhauer, I. Pogorelsky, K. Kusche, R. Fernow, X. Wang, and Y. Liu, Phys. Rev. Lett. 74, 546 (1995).

[21] D. Haberberger, S. Tochitsky, and C. Joshi, Opt. Express 18, 17865 (2010). 